\title{
A vascularized emergency trauma amputation simulator for surgical skills training
}

\author{
Abhishek Chandra, ${ }^{a}, *$ Lauren P. Harvey, ${ }^{a}$ Sahand Fardi, ${ }^{b}$ Eric J. Krohn, ${ }^{a}$ Shumaila Anwer ${ }^{c}$ and James V. Harmon ${ }^{a}$ \\ ${ }^{a}$ Department of Surgery, University of Minnesota, 516 Delaware St. SE, Minneapolis, MN 55455, USA; ${ }^{b}$ University of California, \\ Los Angeles, 405 Hilgard Avenue, Los Angeles, CA 90095, USA; ${ }^{c}$ College of Medicine, University of Central Florida, 6850 Lake \\ Nona Blvd, Orlando, FL 32827, USA
}

${ }^{*}$ Corresponding author at: 2600 University Ave. SE. Unit 205, Minneapolis, MN 55414, USA. Email: chand394@umn.edu

Date accepted for publication: 8 June 2021

\section{Abstract}

Background: We describe the development of a low-cost lower-extremity amputation model enhanced with simulated pulsatile vasculature (SPV). The vascularized emergency trauma amputation simulator (VETAS) permits students to practice clinical decision-making, prepping and draping, hemostatic knot-tying, and lower-extremity amputation. SPV was achieved using a microcontroller, regulating the flow of artificial blood through a bypass flow system, preventing excessive pressure when the SPV is clamped. The SPV system replicates a pulse of 75 beats/min, a pressure of 120/80 $\mathrm{mmHg}$, and a flow rate of $350 \mathrm{~mL} / \mathrm{min}$. We assessed if the model and simulation scenario accomplished our design goals of cost-effective, multidimensional education, with efficient turnover between learners. Methods: A low-cost VETAS was engineered to simulate the tibia and tibial artery using SPV. A simulation scenario was prepared for medical students' training. A pre- and post-training survey was completed by 53 attendees to evaluate the experience. Survey responses were ranked using a Likert scale and analyzed using a Wilcoxon signed-rank test. Results: The VETAS was constructed with inexpensive, readily available materials. The technology allows for efficient, reproducible training. Post-training survey analysis demonstrated statistically significant increases in familiarity with creating a sterile surgical field and with proper instrument handling $(P<0.001)$. An increased sense of confidence regarding lowerextremity surgical anatomy was also confirmed $(P<0.014)$. Conclusion: The VETAS model successfully introduced medical students to the principles of emergency trauma surgery. This novel technology created a cost-effective platform for efficient and effective skills training.

Keywords: surgical simulation; surgical education; amputation; skills training; vascular surgery; trauma

\section{Introduction}

Simulation learning as a tool for surgical training has developed rapidly in its utility and validation. ${ }^{1-3}$ Simulation education offers trainees an opportunity to be immersed in educational concepts, repeatedly practice fundamental techniques, and train for clinical scenarios. In practice, it allows training costs to be kept low and the development of objective skills assessments. One of the key tenets of simulation training is practice; learners benefit from repeated practice sessions with a simulation, especially with feedback, coaching, and training. ${ }^{3-7} \mathrm{~A}$ wide range of factors, of which the COVID-19 pandemic is particularly salient, limit the time trainees have with hands-on clinical activity, therefore the apprenticeship model of surgical training is progressing toward one of competency. Simulation offers standardization of training and avenues for objective learner assessment, feedback, and remediation. ${ }^{8-10}$ However, surgical simulation has less frequently ventured into the recreation of more complex clinical scenarios, such as extremity trauma management, primarily due to logistic, financial, and reusability constraints. ${ }^{11-16}$

Lower extremity trauma is an injury pattern that requires thoughtful oversight led by experienced trauma or orthopedic surgeons. ${ }^{17}$ In a minority of cases, the extent and severity of injury demands a primary amputation at the time of initial trauma assessment and stabilization. ${ }^{18}$ The Western Trauma Association has detailed a current standard algorithm for the evaluation of trauma patients for lower extremity amputations. ${ }^{19}$ The principles of amputation surgery outline the management of skin, vessels, nerves, and bone. Proper management of large vessels includes proximal control and ligation. Intervention for vascular trauma calls for 
the isolation of vessels to ensure hemostasis and avoid postoperative complications such as arteriovenous fistulas or pseudoaneurysm; transection of bone can be approached with a Gigli saw or oscillating-tip power saw. ${ }^{17,20}$

The purpose of this project was to develop a cost-effective surgical model and corresponding simulation for the management of lower extremity amputation. The vascularized emergency trauma amputation simulator (VETAS) incorporated simulated pulsatile vasculature (SPV) using a responsive microcontroller, a specific feature evaluated and proven to enhance training outcomes. ${ }^{21}$ The microcontroller recorded learner performance by measuring simulated model pulse, pressure, and flow rate. The simulator was made of readily available, low-cost materials. Our study was designed to evaluate if this low-cost lower-extremity amputation simulation model would be an effective tool for our medical student learners.

\section{Methods}

\section{Model design}

A low-fidelity model of the lower extremity was constructed using readily available, low-cost materials. Polyvinyl chloride (PVC) piping, $12.7 \mathrm{~mm}$ in diameter, was used to simulate the tibia. Latex tubing with an outer diameter of $8 \mathrm{~mm}$ was attached with a thin strip of cellophane tape to the PVC, simulating the anterior tibial artery. A simple mannequin was used to represent the patient. The tibia and arterial structures were funneled down a larger PVC pipe through the mannequin's shoulder to represent the right leg vasculature and bone structure. Synthetic material, simulating skin, fat, and muscle layers, surrounded the PVC tibia and latex tubing. Commercially available synthetic blood (Nasco, Fort Atkinson, WI) was used for the simulation. Learners only interacted with the simulated anatomic structures; the larger PVC tubing was used solely for structural support and was not visible to trainees.

Simulated pulsatile flow through the vasculature was accomplished by a $12 \mathrm{~V}$ power-supply system consisting of a selfpriming pump motor, pressure transducer, flow meter, and UNO R3 microcontroller (Arduino) (Fig. 1). Pulsatile flow of 75 beats per minute and a flow rate of $300-400 \mathrm{~mL}$ per minute were regulated by a metal oxide silicon field effect transistor (MOSFET) programmed to vary the fluid dynamics. This allowed for authentic pulsatile "bleeding" of the patient's extremity if the procedural steps were not performed correctly. Flow and pressure were monitored using the microcontroller, which that received input from the flow meter and pressure transducer at regular intervals. The blood pressure during pulsatile flow was monitored via the pressure transducer located upstream of the mock vasculature and was set at 120/80 $\mathrm{mmHg}$.

An algorithm was written for the microcontroller that allowed the pulsatile flow to be maintained at 75 beats per minute and the power input to be adjusted to the motor to maintain the fluid pressure near $120 / 80 \mathrm{mmHg}$ during pulsatile flow. Once the mock vasculature was unclamped, the

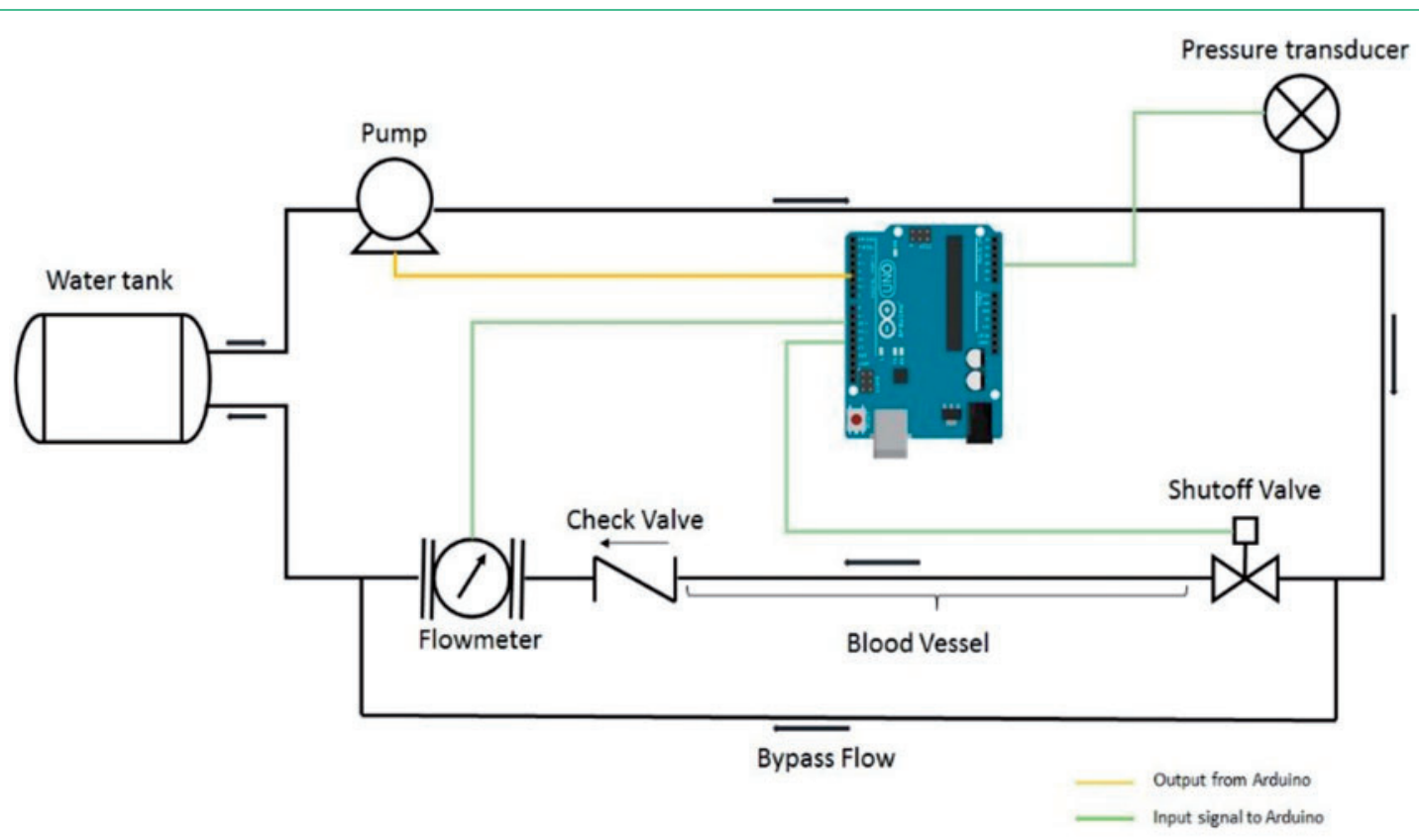

Figure 1. Schematic diagram of the simulated pulsatile flow set-up detailing the Arduino feedback system. 
microcontroller algorithm would switch flow to a steady rate between 0.3 and $0.4 \mathrm{~L} / \mathrm{min}$

\section{Initial simulator evaluation}

Fifty-three medical students from the University of Minnesota Medical School participated in the simulation during a surgical skills workshop. Medical students, in groups of four to five, were instructed on the basic approach to managing trauma (ABCs) including airway assessment and protection, breathing and ventilation assessment, and evaluation of circulation. Learners were introduced to the VETAS and the clinical scenario, a mangled extremity due to a severe crush injury and fracture of the distal tibia. Exploration of the wound revealed complete disruption of the nerves and arteries supplying the distal leg and foot. They were then guided through the appropriate care of the simulated patient by two physician instructors. Proper navigation of the simulation exercise included stabilization of the patient, starting intravenous fluids, evaluating the appropriateness of amputation, prepping and draping, proper surgical exposure through the tissue, proximal vessel ligation, and use of the Gigli saw. The simulated pulsatile vascular system enhanced the fidelity of the simulation by requiring urgent management of an actively bleeding vessel. Learners were guided in clamping the simulated arterial vessel proximally and distally, transecting the vessel, and tying hemostatic knots. The use of readily available PVC piping, latex tubing, and artificial blood provided opportunities for multiple trainees to practice in each rotation. As each trainee completed the guided scenario, the next segments of tibia and artery were easily advanced through the larger PVC housing and prepared for the next learner (Fig. 2). Our model offered a very realistic knot-tying assessment; if learners successfully controlled the artificial bleeding with their suture ligature, they met the knot-tying criteria.

To evaluate the efficacy and utility of the simulator and simulation, a 13-question survey was administered before and after the event. Six of these questions were specific to the amputation simulation. These questions evaluated the participants' comfort with the surgical techniques, use of the Gigli saw, familiarity with lower extremity anatomy, prepping and draping patients, and ability to identify and handle surgical instruments. Responses were ranked using a 5-point Likert scale (Table 1).

\section{Results}

Our team designed and built a low-cost lower extremity simulator that was effective in achieving our training goals for a surgical skills workshop (Fig. 3). The final cost of the lower-extremity simulator was $\$ 98$ (US dollars) (Table 2). On average, trainees spent $20 \mathrm{~min}(2-5 \mathrm{~min}$ for instructor feedback) at the lower-extremity amputation station. Simulation testing confirmed that the SPV device is an effective tool in acquainting trainees with the challenges of lower extremity amputation, including knowledge of anatomy and introducing students to basic surgical techniques. All 53 of the workshop attendees completed the experience

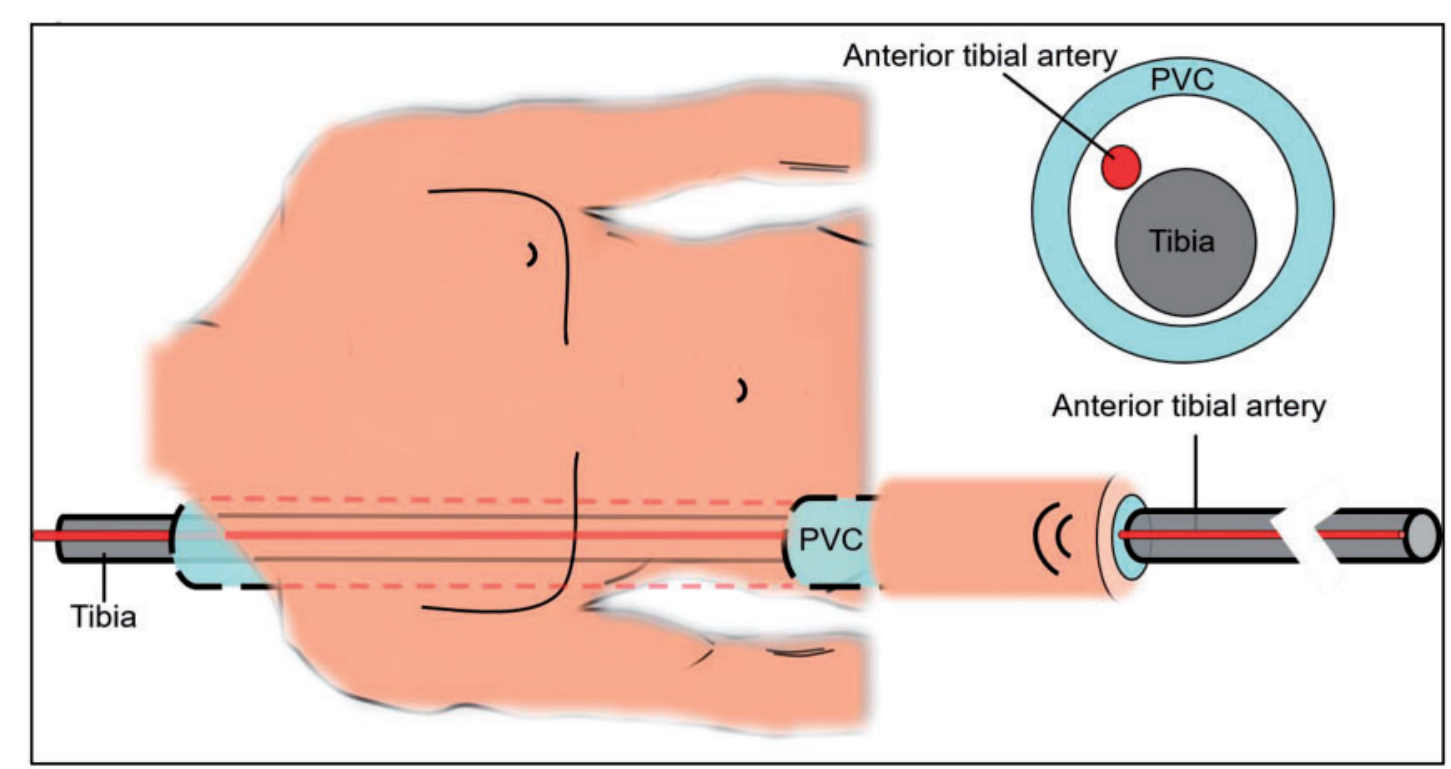

Figure 2. Schematic representation of the simulated vasculature and bone found in the simulated patient, detailing how the VETAS could be used for multiple subsequent learners. 
Table 1. Five-point Likert scale ranked responses to the general workshop and amputation-station-specific questionnaire ( $\mathrm{n}=53$ )

\begin{tabular}{|c|c|c|c|c|c|c|}
\hline & \multicolumn{6}{|l|}{ Likert scale (0-5) } \\
\hline & $\begin{array}{l}\text { Very uninterested, } \\
\text { unfamiliar, } \\
\text { uncomfortable }\end{array}$ & 1 & 2 & 3 & 4 & $\begin{array}{l}\text { Very interested, } \\
\text { familiar, } \\
\text { comfortable }\end{array}$ \\
\hline How would you currently rate your interest in surgery? & 0 & 0 & 1 & 3 & 15 & 34 \\
\hline How prepared are you for surgery clerkships? & 9 & 24 & 10 & 6 & 2 & 2 \\
\hline How comfortable are you with your knot-tying skills? & 2 & 10 & 12 & 15 & 10 & 4 \\
\hline How comfortable are you with sterile techniques in the operating room? & 0 & 7 & 11 & 15 & 16 & 4 \\
\hline How comfortable are you in prepping and draping in the operating room? & 1 & 9 & 9 & 23 & 10 & 1 \\
\hline How comfortable are you in identifying and handling surgical instruments? & 11 & 3 & 14 & 18 & 7 & 0 \\
\hline How familiar are you with anatomy of the lower extremity? & 3 & 6 & 13 & 24 & 3 & 4 \\
\hline How comfortable are you in using the Gigli saw? & 2 & 12 & 9 & 15 & 10 & 5 \\
\hline
\end{tabular}

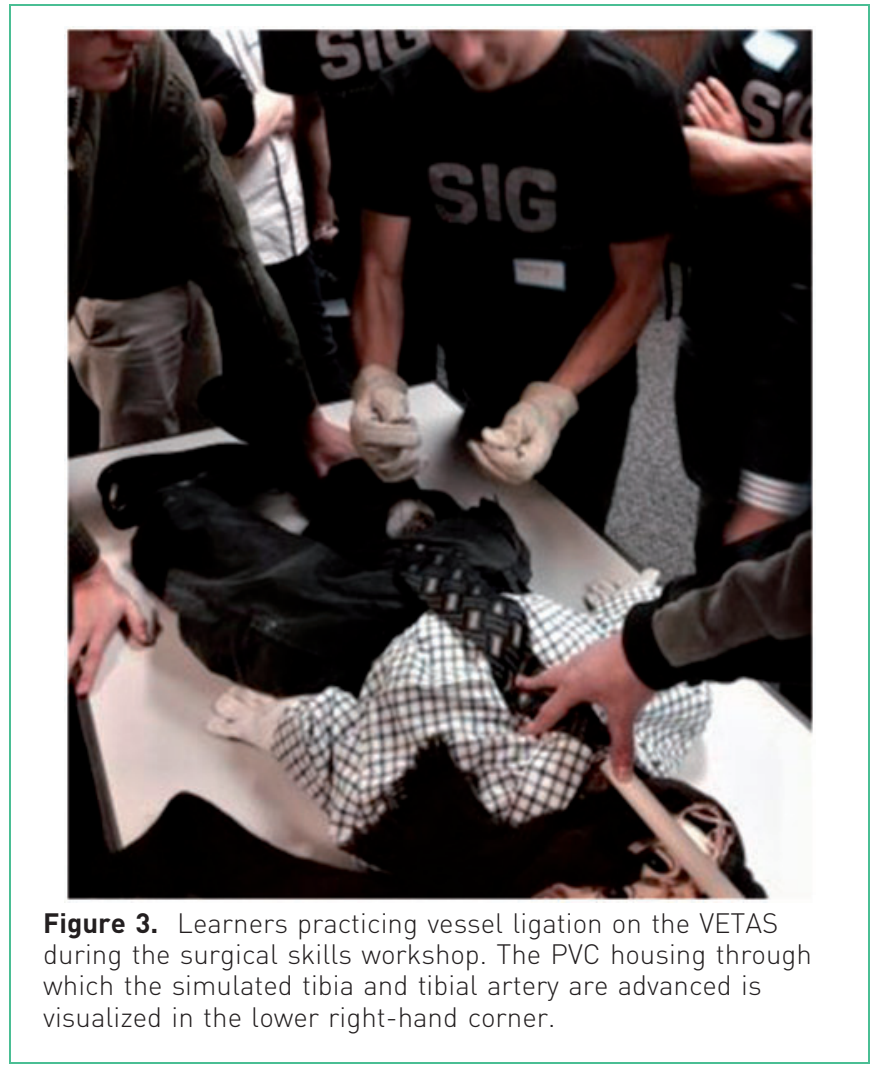

survey (Table 1). Of the five activities presented at the workshop, the simulation of lower extremity amputation received the highest acclaim. Other activities included simulated breast biopsy, bedside ultrasonography, knot-tying skills, and suture training. Learners reported being more familiar with lower extremity surgical anatomy $(P<0.014)$ and better acquainted with surgical techniques $(P<0.001)$, including the use of a Gigli saw $(P<0.001)$. The survey suggested that the model and simulation had utility beyond the clinical
Table 2. Cost breakdown of the simulated pulsatile vasculature system

\begin{tabular}{ll}
\hline Component & $\begin{array}{l}\text { Cost } \\
\text { (US\$) }\end{array}$ \\
\hline Power supply & 8.00 \\
Pump motor (self-priming) & 22.00 \\
Pressure transducer & 1.50 \\
Flow meter & 22.00 \\
Shutoff valve (normally open) & 0.50 \\
Check valves & 6.50 \\
Microcontroller (Arduino UNO R3) & 22.00 \\
Tubing (PVC) & 7.00 \\
Mock vasculature (latex) & 3.00 \\
Bypass flow tubing (latex and vinyl) & 5.00 \\
MOSFET (IRFZ34N) & 0.50 \\
Total & 98.00 \\
\hline
\end{tabular}

scenario; learners also reported feeling more familiar with prepping patients in the operating room $(P<0.001)$ and more comfortable handling surgical instruments $(P<0.001)$. The pulsatile bleeding of the simulator was helpful to achieve the "willing suspension of disbelief" during this scenario, and the use of the Gigli saw created an engaging and active hands-on experience for the learners.

\section{Discussion}

Lower extremity amputation is a complex procedure that is associated with a significant perioperative morbidity and mortality. ${ }^{20,21}$ It requires a balance of two primary goals: immediate removal of devascularized, damaged, or dysfunctional tissue, and reconstruction. It is valuable for trainees 
to have early exposure to amputation procedures to bolster early surgical skill acquisition in the context of simulated patient care. ${ }^{22}$ Simulation using synthetic models is a wellestablished, effective tool to develop technical skills transferable to an operating room for a surgical trainee. ${ }^{25-29}$ Simulation training has become particularly important because hands-on clinical time is limited in the COVID19 era. For medical students, immersion in a clinical scenario is likely to promote durable retention of skills through increased global alertness and engagement in the mock trauma training session..$^{30,31}$

Many training and assessment modalities are often limited by cost and sustainability (Table 3). ${ }^{32,33}$ The use of animal or cadaveric models are alternatives to live patient training, but are expensive and require diligent ethical considerations. ${ }^{34}$ Lower-fidelity models of amputation have been described, but do not offer the immersion of prepping and draping, simulated pulsatile flow, or true-to-life use of the Gigli saw. The benefits of low- versus high-fidelity simulators are poorly defined, but our low-cost, moderate-tohigh-fidelity simulator promises to effectively represent the operative surgical procedure. ${ }^{31,35}$ We believe this model, or one similar to it, will be useful in settings with limited financial resources and a large number of trainees. For novice learners such as medical students, low-fidelity simulation has been demonstrated to be an effective tool for the development of fundamental conceptual understanding of medical procedures and for basic skill acquisition. This simulator is ideal for student learners given that it focuses on fundamental skills training while maintaining an engaging scenario to enhance learning retention. Furthermore, the benefits of participating in trauma simulation are insights relevant to future trainees in a wide variety of specialties that includes family practice, emergency medicine, general surgery, orthopedics, plastic surgery, rehabilitation medicine, and vascular surgery.

Table 3. Comparison of the costs of the in-house lower extremity simulator with commercially available options

\begin{tabular}{ll}
\hline Simulator & $\begin{array}{l}\text { Cost } \\
\text { (US\$) }\end{array}$ \\
\hline $\begin{array}{l}\text { In-house design: VETAS } \\
\text { Simulaids Amputated Bleeding Leg, Nasco, Wisconsin }\end{array}$ & 248.00 \\
$\begin{array}{l}\text { Anatomy Lab Amputated and Trauma Bleeding Leg, } \\
\quad \text { Anatomy Warehouse, Illinois }\end{array}$ & 550.00 \\
$\begin{array}{l}\text { Simulaids Xtreme Trauma Bleeding, Nasco, Wisconsin } \\
\text { Anatomy Bequest Program Cadaveric }\end{array}$ & 757.60 \\
$\quad \begin{array}{l}\text { Preparation, Minnesota } \\
\text { Partial Leg Amputation Simulator, Nasco, Wisconsin }\end{array}$ & 1400.00 \\
\hline
\end{tabular}

A benefit of utilizing simulation-based training to coach foundational surgical skills is the opportunity to mediate the stress of the surrounding scenario; simulation offers curated learning environments that can maximize skill acquisition and retention. Stresses experienced in a clinical setting cannot be controlled, hindering the ability to optimally teach and learn. Harvey et al. ${ }^{35}$ identified the stress levels experienced by emergency medicine and general surgery residents in traumatic simulations with different levels of stress. After participating in both simulations, the residents demonstrated higher subjective stress and cortisol levels, significantly poorer clinical performance, and felt that their resources did not meet the demands of the high stress scenario compared with the low stress scenario. These results reveal stress as a detrimental factor to the fulfillment of the clinical duties of medical professionals. The plasticity of simulation is crucial in achieving a level of "good stress" for learners to practice the fundamental techniques in an environment that mediates the impact of stress on performance, mastery, and clinical application. ${ }^{35,36}$

One of the perceived drawbacks of simulation education is that of fidelity in relation to the clinical equivalent. ${ }^{31}$ Our model works to address these limitations through a high-fidelity pump system that replicates steady and pulsatile blood flow. Fidelity can also be credited to the simulated clinical scenario, which enhanced immersion and offered opportunities to evaluate learners' broader knowledge and composure under pressure. Utilizing an integrated simulator within an immersive simulation is useful in developing foundational surgical skills, without disrupting the medical students' learning.

Future enhancements of this model and the training experience will aim to create a more dynamic simulation for advanced learners. These enhancements would include running the simulation with variable pulsatile flow pressures, vital sign instability alarms, and additional learner feedback in response to inadequate vascular ligation. Clinical scenario and simulation enhancements might also include a liquidcrystal display to project vitals and recreate authentic flow characteristics. Incorporating these future enhancements may improve skill retention, learner confidence, and technical proficiency by allowing learners to prepare for potential intraoperative complications in the operating room. In addition, implementing a system to record and assess student performance through the time spent completing the procedure and amount of blood lost would allow for better academic evaluation and progress tracking. We aim to create a modular simulator based on pre-formed cartridges of the tibia, vasculature, skin, and adipose tissue to improve the fidelity of the simulator, while abiding by the principles of economy, reusability, and portability. We aim to enhance our 
guided debriefings to further discuss surgical decisionmaking and comparing patient outcomes after emergent lower extremity amputation versus limb salvage procedures. We plan to demonstrate design verification, face validity, construct validation, parameter sensitivity, and predictive validity for the simulator and simulation scenario.

In this study we describe a low-cost amputation model and simulation scenario evaluated as a tool to introduce medical students to the rationale behind trauma evaluation, resuscitation, and urgent amputation. Participant survey results demonstrate that the model was educational, valuable, challenging, and accurately simulated the performance of lower extremity amputation. We were successful in creating a lowcost vascularized model of the lower extremity. Integrating psychomotor skills training in the simulation scenario authenticated the training experience as students achieved vascular control and used the handheld Gigli saw. This new simulator accommodates rapid turnover and repeated practice among learners. The technology demonstrates promise for effective training on obtaining hemostatic control, performing suture ligature of simulated vessels, and the transection of the simulated bone.

\section{Conflict of interest}

The authors declare that they have no affiliations with or involvement in any organization or entity with any financial interest or non-financial interest in the subject matter or materials discussed in this manuscript.

\section{Acknowledgments}

We thank all our colleagues from the University of Minnesota who provided insight and expertise that greatly assisted the research. Special thanks to Alex Charboneau, Alexander DiBartolomeo, Peter Kernahan, Elizabeth Smith, and Victor Vakayil for their assistance and expertise for this project. Finally, we would like to express our gratitude to the physician educators and members of the University of Minnesota Surgical Interest Group for their work in organizing and executing the skills workshop. This research effort was supported by the William W. Harmon Fund for Surgical Research and Education and the James Lord Surgical Education Fund and Mic Lord Surgical Education Fund.

\section{References}

1. Goldenberg M, Lee JY. Surgical education, simulation, and simulators-updating the concept of validity. Curr Urol Rep 2018; 19(7): 52. https://doi.org/10.1007/s11934-018-0799-7.
2. Dunkin B, Adrales GL, Apelgren K, Mellinger JD. Surgical simulation: a current review. Surg Endosc 2007; 21(3): 357366. https://doi.org/10.1007/s00464-006-9072-0.

3. Nabavi A, Schipper J. Op.-Simulation in der Chirurgie [Simulation in surgical training]. HNO 2017; 65(1): 7-12 [in German]. https://doi.org/10.1007/s00106-016-0248-1.

4. Yanagawa B, Ribeiro R, Naqib F, Fann J, Verma S, Puskas JD. See one, simulate many, do one, teach one: cardiac surgical simulation. Curr Opin Cardiol 2019; 34(5): 571-577. https://doi.org/10.1097/HCO.0000000000000659.

5. Saleem HY, AlJamal Y, Prabhakar N, Baloul M, Balachandran P, Farley D. Low-cost materials yield high resolution assessment of anatomic knowledge in surgical residents. Surgery 2019; 165(6): 1088-1092. https://doi.org/10.1016/j.surg.2019. 01.017 .

6. AlJamal Y, Prabhakar N, Rivera M, McKenzie T, Stulak J, Heller S, et al. A simulation-based selection process for trying to identify medical students who will become outstanding general surgery residents. Am J Surg 2020; 220(1): 76-82. https://doi.org/10.1016/j.amjsurg.2019.10.050.

7. AlJamal Y, Ali SM, Ruparel RK, Brahmbhatt RD, Yadav S, Farley DR. The rationale for combining an online audiovisual curriculum with simulation to better educate general surgery trainees. Surgery 2014; 156(3): 723-728. https://doi.org/10. 1016/j.surg.2014.04.049.

8. Dunn W, Dong Y, Zendejas B, Ruparel R, Farley D. Simulation, mastery learning and healthcare. Am J Med Sci 2017; 353(2): 158-165. https://doi.org/10.1016/j.amjms.2016. 12.012 .

9. Chandra A, Rajesh A, Backstrom C, Farley DR. Training, testing and remediating: the importance of simulation for surgical trainees. ANZ J Surg 2019; 89(11): 1364-1365. https://doi.org/10.1111/ans.15274.

10. Cooke JM, Rooney DM, Fernandez GL, Farley DR. Simulation center best practices: a review of ACS-accredited educational institutes' best practices, 2011 to present. Surgery 2018; 163(4): 916-920. https://doi.org/10.1016/j.surg.2017.11.004.

11. Schneider E, Schenarts PJ, Shostrom V, Schenarts KD, Evans CH. "I got it on Ebay!": cost-effective approach to surgical skills laboratories. J Surg Res 2017; 207: 190-197. https://doi. org/10.1016/j.jss.2016.08.017.

12. Ellinas H, Denson K, Simpson D. Low-cost simulation: howto guide. J Grad Med Educ 2015; 7(2): 257-258. https://doi. org/10.4300/JGME-D-15-00082.1.

13. Hammond J. Simulation in critical care and trauma education and training. Curr Opin Crit Care 2004; 10(5): 325-329. https://doi.org/10.1097/01.ccx.0000140950.47361.c9.

14. Bowles C, Canuto D, Teran J, Dutson E, Plurad D, Eldredge J, et al. Current methods and advances in simulation of hemorrhage after trauma. Am Surg 2017; 83(10): 1137-1141. https://doi.org/10.1177/000313481708301025. 
15. Flott K, Gage W, Batey M, Redhead J, Darzi A. Improving patient safety culture through low cost innovation [abstract]. J Surg Simul 2018; A5. https://doi.org/10.1102/2051-7726.2018.A005.

16. Yon J, Mentzer CJ, Adam BL, Young L. A low-cost, non-biologic, thoracentesis and thoracostomy simulator. J Surg Simul 2015; 2: 18-21. https://doi.org/10.1102/2051-7726.2015.0004.

17. Higgins TF, Klatt JB, Beals TC. Lower Extremity Assessment Project (LEAP)-the best available evidence on limb-threatening lower extremity trauma. Orthop Clin North Am 2010; 41(2): 233-239. https://doi.org/10.1016/j.ocl.2009.12.006.

18. Schirò GR, Sessa S, Piccioli A, Maccauro G. Primary amputation vs limb salvage in mangled extremity: a systematic review of the current scoring system. BMC Musculoskelet Disord. 2015; 16(1): 1-7. https://doi.org/10.1186/s12891-015-0832-7.

19. Feliciano DV, Moore EE, West MA, Moore FA, Davis JW, Cocanour CS, et al. Western Trauma Association critical decisions in trauma: evaluation and management of peripheral vascular injury, part II. J Trauma Acute Care Surg 2013; 75(3): 391-397. https://doi.org/10.1097/TA.0b013e3182994b48.

20. Leech C, Porter K. Man or machine? An experimental study of prehospital emergency amputation. Emerg Med J 2016; 33(9): 641-644. https://doi.org/10.1136/emermed-2015-204881.

21. Min SK. Big Challenges in training young vascular surgeons. Vasc Specialist Int 2019; 35(3): 119. https://doi.org/10.5758/vsi. 2019.35.3.119.

22. Pourghaderi P, Yuquimpo KM, Guetter CR, Mansfield L, Park HS. Outcomes following lower extremity amputation in patients with diabetes mellitus and peripheral arterial disease. Ann Vasc Surg 2020; 63: 259-268. https://doi.org/10.1016/j. avsg.2019.08.084.

23. Gabel J, Jabo B, Patel S, Kiang S, Bianchi C, Chiriano J, et al. Vascular quality initiative. Analysis of patients undergoing major lower extremity amputation in the vascular quality initiative. Ann Vasc Surg 2018; 46: 75-82. https://doi.org/10. 1016/j.avsg.2017.07.034.

24. Zendejas B, Cook DA, Bingener J, Huebner M, Dunn WF, Sarr MG, et al. Simulation-based mastery learning improves patient outcomes in laparoscopic inguinal hernia repair: a randomized controlled trial. Ann Surg 2011; 254(3): 502511. https://doi.org/10.1097/SLA.0b013e31822c6994.

25. Cannon-Bowers JA. Recent advances in scenario-based training for medical education. Curr Opin Anaesthesiol 2008; 21(6): 784-789. https://doi.org/10.1097/ACO.0b013e3283184435.

26. Scott DJ, Pugh CM, Ritter EM, Jacobs LM, Pellegrini CA, Sachdeva AK. New directions in simulation-based surgical education and training: validation and transfer of surgical skills, use of nonsurgeons as faculty, use of simulation to screen and select surgery residents, and long-term follow-up of learners. Surgery 2011; 149(6): 735-744. https://doi.org/10. 1016/j.surg.2010.11.010.

27. Carden AJ, Salcedo ES, Leshikar DE, Utter GH, Wilson MD, Galante JM. Randomized controlled trial comparing dynamic simulation with static simulation in trauma. J Trauma Acute Care Surg 2016; 80(5): 748-754. https://doi.org/10.1097/TA. 0000000000001012.

28. Massoth C, Röder H, Ohlenburg H, Hessler M, Zarbock A, Pöpping DM, et al. High-fidelity is not superior to low-fidelity simulation but leads to overconfidence in medical students. BMC Med Educ 2019; 19(1): 29. https://doi.org/10. 1186/s12909-019-1464-7.

29. Burke CR, Mokadam NA. Repetition is the mother of skill. J Thorac Cardiovasc Surg 2018; 155(4): 1694. https://doi.org/10. 1016/j.jtcvs.2017.12.096.

30. Motola I, Devine LA, Chung HS, Sullivan JE, Issenberg SB. Simulation in healthcare education: a best evidence practical guide. AMEE Guide No. 82. Med Teach 2013; 35(10): e15111530. https://doi.org/10.3109/0142159X.2013.818632.

31. Evans L, Taubert M. State of the science: the doll is dead: simulation in palliative care education. BMJ Support Palliat Care 2019; 9(2): 117-119. https://doi.org/10.1136/bmjspcare2018-001595.

32. Bing EG, Parham GP, Cuevas A, Fisher B, Skinner J, Mwanahamuntu $\mathrm{M}$, et al. using low-cost virtual reality simulation to build surgical capacity for cervical cancer treatment. J Glob Oncol 2019; 5: 1-7. https://doi.org/10.1200/JGO.18. 00263.

33. Hauer T, Schneider K, Mayer D, Huschitt N, Lieber A, Willy C. Technische Trainingsmodelle für die notfallchirurgische Ausbildung: Bedarf, Status quo und Potenzial [Human patient simulators for training in emergency surgery: needs, status quo and potential]. Unfallchirurg 2019; 122(6): 452-463 [in German]. https://doi.org/10.1007/s00113-019-0663-3.

34. Harvey A, Bandiera G, Nathens AB, LeBlanc VR. Impact of stress on resident performance in simulated trauma scenarios. J Trauma Acute Care Surg 2012;72(2): 497-503. https://doi. org/10.1097/ta.0b013e31821f84be.

35. Sachedina AK, Blissett S, Remtulla A, Sridhar K, Morrison D. Preparing the next generation of code blue leaders through simulation: what's missing? Simul Healthc 2019; 14(2): 77-81. https://doi.org/10.1097/SIH.0000000000000343.

36. Wier GS, Tree R, Nusr R. Training effectiveness of a wide area virtual environment in medical simulation. Simul Healthc 2017; 12(1): 28-40. https://doi.org/10.1097/SIH.0000000000 000207. 